competitiveness especially of small and medium sized enterprises, where OSD-incidence peaks. For affected individuals, the chronic course of OD may result in job loss, precarious or unemployment. A joint coordinated approach to patient management as well as standardization of diagnostics, therapy, protective equipment as well as workers' education is lacking in Europe and other parts of the World. Important sustainable prevention strategies applied in various countries will be presented during this session.

\section{8 a OSD PREVENTION: WHY? A EUROPEAN AND GLOBAL PERSPECTIVE}

Swen Malte John. Dept. Dermatology, Environmental Medicine, Health Theory, University of Osnabrueck, Germany

\subsection{6/oemed-2018-ICOHabstracts.257}

Introduction In Europe, occupational skin diseases (OSD) mainly irritant and allergic contact dermatitis of the hands constitute currently up to $40 \%$ of all work-related illnesses causing extensive suffering for affected workers. Due to emerging new workplace hazards and demographic change OSD are likely to increase over the next years. The annual costs incurred by OSD are estimated to exceed by far 5 billion $€$ in the EU due to medical treatment, sick leave and loss of productivity.

Results Recent studies have demonstrated that dermatological intervention can save OSD patients' health and jobs, and avoid individual suffering as well as reduce costs for society. A longitudinal study carried out in Germany with severely OSD affected workers showed that due to an interdisciplinary in-patient prevention scheme $80 \%$ of patients were still working 3 years after the measure and sick leave was sustainably reduced (total cohort: $N=1,409$ ). Similar results were obtained from a randomized nationwide follow up study of initial cases of contact dermatitis receiving outpatient skin protection seminars and regular outpatient treatment by the local dermatologists $(\mathrm{N}=1,600)$. However, as yet, insurance systems in many countries do neither enable specific dermatological intervention nor specific preventive measures. Since 2010 the EADV 'healthy skin @work' campaign aims at raising awareness at the primary prevention level, and also to improve options for medical care for affected individuals, and coordinate scientific efforts. This dermatological initiative is also an official partner of the 'Healthy workplaces campaign' by the European Agency for Safety and Health at Work (EU OSHA).

Discussion In various European countries OSD specific centers have been or are being implemented (e.g. Austria and Denmark) geared at applying specifically tailored measures for OSD patients. In Germany on the other hand, where since 2009 the 'Week of Occupational skin Diseases (WOOD)' has been carried out, a 30\% increase of OSD notifications could be observed, which will help tackle the under-reporting. At the same time, costs for job-retraining have substantially decreased due to earlier and more effective preventive intervention. 1618b THE OCCUPATIONAL DISEASE ACTION PLAN: ONTARIO, CANADA TAKES A STEP FORWARD

Linn Holness. Centre for Research Expertise in Occupational Disease, University of Toronto and St Michael's Hospital, Ontario, Canada

\subsection{6/oemed-2018-ICOHabstracts.258}

Introduction Government legislation, regulation and policy are important components of workplace prevention. While many jurisdictions have legislation and regulations that provide direction for workplace prevention, it is also important the government promotes workplace prevention in priority areas. In Ontario the focus has traditionally been on accidents and ergonomic issues. In 2016 the Province of Ontario, Canada initiated an Occupational Disease Action Plan (ODAP) to move the prevention of occupational disease into priority focus. The objective of this session is to describe the process leading to the development and implementation of the ODAP with respect to skin allergens and irritants.

Methods The Prevention Office of the Ontario Ministry of Labour held a meeting bringing together of occupational disease research and data experts and occupational health and safety (OHS) system partners to review and recommend where prevention efforts should be focused. Two lists of potential exposure and disease priorities were developed. A Reference Group was formed of OHS system partners that created a propriety ranking of areas for focus. An Implementation Team and Working Groups were formed to carry each priority area forward.

Results The five priority areas identified included general occupational disease awareness and skin and lung allergens and irritants. A working group for Allergens and Irritants is initially identifying priority allergens and irritants and will then move forward with awareness, resource and training development.

Discussion The development and implementation of the ODAP will see increased government and OHS system partner focus on occupational disease including skin allergens and irritants. Following prioritization of key skin allergens and irritants, resources and training opportunities will be reviewed and gaps filled. In addition, Ministry of Labour operations staff will ensure that the ODAP priorities are included in inspections and other activities.

\section{$1618 \mathrm{C}$ EFFECTIVENESS OF SECONDARY PREVENTION FOR OCCUPATIONAL DERMATITIS: THE EXPERIENCE IN TRIESTE HOSPITALS}

Francesca Larese Filon. Unit of Occupational Medicine, Dept. of Medical Sciences, University of Trieste, Italy

\subsection{6/oemed-2018-ICOHabstracts.259}

Introduction Primary and secondary prevention for occupational contact dermatitis is a challenge for the occupational physician: educational training and the avoidance of contact with irritant and allergic substances permits a reduction of incidence cases and days of work lost for a disease that is the third in frequency among workers in industrialized countries. Methods Workers with a diagnosis of occupational hand dermatitis were invited to a training course on prevention of skin 
dermatitis, their skin condition were evaluated by a dermatologist/allergologist and by TEWL (trans-epidermal water loss) measurements. Information on skin disease, occupational and non-occupational exposure were recorded using a standardized questionnaire. Workers participated to a second control after 3 months, to verify the effectiveness of the training.

Results 80 workers with an occupational hand dermatitis accepted to participate to a training course and 62 (77.5\%) completed the 3 months follow-up. Symptoms improved after the training for the $73 \%$ of subjects that adhered to suggestions and followed recommendations and for the $38 \%$ of workers that partially followed recommendations $(p<0.01)$. TEWL values improved at the end of follow-up, confirming the positive effect of protective measurements suggested on skin barrier function.

Discussion Our intervention was effective, leading to a reduction in clinical signs of dermatitis in people that strictly adhere to recommendations, mainly on cleaning habits and skin cream use. The educational intervention permitted to improve skin condition in a short-term follow-up. More efforts are needed to train workers on skin protection procedures.

\section{8d SCIN TRIAL (SKIN CARE INTERVENTION IN NURSES)}

Ira Madan. Guy's and St Thomas NHS Foundation Trust, London, UK

\subsection{6/oemed-2018-ICOHabstracts.260}

Introduction The high prevalence of hand dermatitis in nurses is attributed to frequent hand washing with soap and infrequent use of hand moisturisers. We tested the hypothesis that a behavioural change programme (BCP) coupled with hand moisturisers, can reduce the prevalence of hand dermatitis in at-risk nurses over a 12 - month period.

Methods We conducted a cluster randomised design at 35 sites. We recruited two groups of at-risk nurses:

a. first year student nurses with a history of atopic tendency;

b. intensive care unit (ICU) nurses.

The BCP was offered to participants at intervention plus sites only and participants were asked to form implementation intentions for performing each hand care behaviour in the workplace. Student nurses were provided with personal supplies of hand creams and ICU nurses encouraged to make use of the moisturisers on the wards. All participants, including those at intervention light sites, were provided with an advice leaflet on optimal hand care. Participants had their hands photographed at baseline and 12 months and these were objectively assessed by a dermatology research nurse and two dermatologists. We also measured changes in hand dermatitis, beliefs and behaviours and collected health economic data.

Results Overall the results indicate that the intervention had a positive (but not statistically significant at 5\% level) effect in reducing the prevalence of hand dermatitis compared to usual care and had a positive effect in changing behaviours and beliefs associated with dermatitis prevention. The BCP was also found to be of low cost to implement. (Please note, final results were not available by the abstract submission date but will be provided to the Secretariat in due course).

Discussion A hand-dermatitis prevention BCP plus optimal provision of hand moisturisers has been shown to be effective in reducing hand dermatitis in nurses. 1618e MINIMUM STANDARDS ON PREVENTION, DIAGNOSIS AND TREATMENT OF OCCUPATIONAL AND WORKRELATED SKIN DISEASES IN EUROPE - POSITION PAPER OF THE COST ACTION STANDERM (TD 1206)

${ }^{1} J o s e ́$ Hernán Alfonso, ${ }^{2}$ Patricia Weinert. ${ }^{1}$ Dept. of Occupational Medicine and Epidemiology, National Institute of Occupational Health, Oslo, Norway; ${ }^{2}$ Institute for interdisciplinary Dermatological Prevention and Rehabilitation (iDerm), Germany

\subsection{6/oemed-2018-ICOHabstracts.261}

Introduction Skin diseases constitute up to $40 \%$ of all notified occupational diseases in most European countries, predominantly comprising contact dermatitis, contact urticaria, and skin cancer. While insufficient prevention of work-related skin diseases (WRSD) is a top-priority problem in Europe, common standards for prevention of these conditions are lacking.

Objective To develop common European standards on prevention and management of WRSD and occupational skin diseases (OSD). Method Consensus amongst ca. 100 experts within occupational dermatology was achieved with regard to the definition of minimum evidence-based standards on prevention and management of WRSD/OSD (Delphi Technique).

Results By definition, WRSDs/OSDs are (partially or fully) caused by occupational exposure. The definition of OSD sensu stricto additionally includes diverging national legal requirements, with an impact on registration, prevention, management, and compensation. With the implementation of the classification of WRSD/OSD in the International Classification of Diseases (ICD) 11th Revision in future, a valid surveillance and comparability across countries will be possible. Currently, WRDS and OSD are still under-reported. Depending on legislation and regulations, huge differences exist in notification procedures in Europe, although notification is crucial to prevent chronic and relapsing disease. Facilities for early diagnosis, essential for individual patient management, should be based on existing guidelines and include a multidisciplinary approach. Patch testing is essential if contact dermatitis persists or relapses. Workplace exposure assessment of WRSD/OSD requires full labelling of product ingredients on material safety data sheets helping to identify allergens, irritants and skin carcinogens. Comparable standards in primary, secondary and tertiary prevention must be established in Europe to reduce the burden of WRSD/OSD in Europe.

Conclusion The adoption of common European standards on prevention of WRSD/OSD will contribute to reduce the incidence of OSD and their socio-economic burden.

\section{REFERENCE}

1. JEADV 2017;31(Suppl 4):1-13.

\section{Education and Training}

\section{HOW HEALTHY ARE MALE HEALTH SCIENCES STUDENTS AT IMAM ABDULRAHMAN BIN FAISAL UNIVERSITY?}

Sultan Al-Otaibi, Abullah Almutahhar, Taha Aljishi, Hussain Aljawad, Adulaiziz Sebiany. Imam Abdulrahman Bin Faisal University, Damam, Saudi Arabia

\subsection{6/oemed-2018-ICOHabstracts.262}

Objectives This study aimed to collect information on the personal health status of health sciences students in Imam Abdulrahman Bin Faisal University. It also aimed to investigate the 